\title{
DISCUSIONES ACTUALES, OPORTUNIDADES Y HORIZONTES EN LOS ESTUDIOS SOBRE LITERACIDADES EN AMÉRICA LATINA
}

\author{
QUESTÓES ATUAIS, OPORTUNIDAdES E PERSPECTIVAS NOS ESTUdOS DE LETRAMENTOS \\ NA AmÉrica Latina \\ Current Issues, Opportunities, and Horizons in Literacy Studies \\ IN LATIN AMERICA
}

\author{
Emilce Moreno Mosquera \\ Doctora en Educación, Universidad \\ Pedagógica Nacional. Profesora \\ e investigadora de la Facultad de \\ Educación, Pontificia Universidad \\ Javeriana, Bogotá, Colombia. \\ Cra. 7 N. $^{\circ}$ 42-27, Universidad \\ Javeriana, Bogotá. \\ moreno-e@javeriana.edu.co

\section{Luanda Sito} \\ Doctora en Lingüística Aplicada, \\ Universidade Estadual de Campinas \\ (UNICAMP). Profesora e investigadora \\ de la Facultad de Educación, \\ Universidad de Antioquia, Medellín, \\ Colombia. \\ Calle 67 N. ${ }^{\circ} 53$-108, Ciudad \\ Universitaria, Universidad de \\ Antioquia, Medellín-Colombia. \\ luanda.soares@udea.edu.co
}

\section{RESUMEN}

Para dar apertura al presente número, las editoras han querido reflexionar sobre algunos principios en los cuales se basan los estudios sobre literacidades y la manera como estos podrían ser relevantes para la educación en América Latina. Los Nuevos Estudios de Literacidad (también llamados Nuevos Estudios de Cultura Escrita) representan un cambio en la comprensión sobre el abordaje y la adquisición de las prácticas letradas en contextos culturales específicos, en oposición a modelos cognitivos dominantes, de carácter más experimental e individualista. Para dar alcance a esta reflexión, se explicará el marco en el que se originó el interés de las editoras por el tema, así como el lugar que tienen el lenguaje, las prácticas de lectura y escritura en los estudios de literacidades, para finalmente plantear unas líneas de acción abiertas y relevantes para la educación en América Latina.

Palabras clave: Nuevos Estudios de Literacidad, NLS, déficit lingüístico y cultural, educación; enseñanza del lenguaje; investigación en literacidades; lectura y escritura; América Latina.

\section{RESUMO}

Para dar início a esta edição, as editoras buscaram refletir sobre alguns princípios que subjazem os estudos sobre letramentos, assim como a maneira como estes poderiam contribuir para a educação na América Latina. Os Novos Estudos de Letramento (também chamados Novos Estudos de Cultura Escrita) representam uma mudança na compreensão sobre a abordagem e sobre a aquisição das práticas letradas em contextos culturais específicos, em oposição aos padrões cognitivos dominantes, de tipo mais experimental e individualista. Para dar conta desta reflexão, se explicará o contexto no qual surgiu o interesse das autoras pela questão, assim como o lugar que tem a linguagem e as práticas de leitura e escrita nos estudos dos letramentos, para finalizar apresentando umas linhas de ação abertas e relevantes para a educação na América Latina.

Palavras chave: Novos Estudos de Letramento, NLS, déficit linguístico e cultural, educação; ensino da linguagem; pesquisa em letramentos; leitura e escrita; América Latina. 


\section{Abstract}

To open this issue, we as guest editors aim to reflect on some fundamentals supporting literacy studies, and how these could be relevant to education in Latin America. New Literacy Studies (also called New Written-Culture Studies) account for a shift in how we understand literacy practices approach and acquisition in given cultural contexts, versus mainstream cognitive models, in a rather experimental and individualistic approach. To accomplish this aim, we will explain how our interest on this topic aroused, and which role language, and reading and writing practices play in literacy studies, to end up suggesting lines of action open and relevant to education in Latin America.

Keywords: New Literacy Studies; NLS; language and cultural deficit; language teaching; research on literacies; reading and writing; Latin America. 


\section{Origen del número}

Este número, sobre Estudios de literacidades en América Latina, ${ }^{1}$ recoge trabajosquesevienendesarrollando en torno al concepto de literacidades en países latinoamericanos. Usamos el concepto en plural para destacar las diferentes prácticas sociales, históricas y culturales que involucran la literacidad como actividad, teniendo en cuenta, con Zavala, que "escribir ya no es solo un producto (lingüístico) o un proceso (cognitivo), sino una práctica situada, social, material, ideológica e histórica” (2011, p. 56), lo que confirma Gee, al señalar que se lee y se escribe de formas específicas, las cuales "están determinadas por los valores y las prácticas de los diferentes grupos sociales y culturales" (2015, p. 36).

En este número se reúnen trabajos sobre diferentes escenarios de la vida, que se enmarcan teórica, epistemológica y metodológicamente en la perspectiva sociocultural de los estudios de literacidad, como denomina Zavala al enfoque desarrollado por Street (1984) bajo el nombre de New Literacy Studies (NLS).

El principal dínamo de esta publicación es la constatación de que este es un campo muy fructífero en diferentes latitudes, pero todavía muy tímido en Colombia. Por esta razón, se propone poner en común los trabajos de investigadores de diferentes países de Latinoamérica, quienes analizan, desde un enfoque sociocultural y crítico, la apropiación de las prácticas de lectura y escritura en diferentes esferas de la actividad humana. Con este objetivo, el diálogo que se teje entre los artículos permite: i) reconocer preguntas de investigación en torno a la lectura y la escritura, desde la perspectiva de los NLS; ii) identificar los campos disciplinares con los cuales se viene dialogando; iii) discutir los aportes de esa perspectiva para los estudios sobre

1 Agradecemos al apoyo de la Facultad de Educación de la Pontificia Universidad Javeriana, a la Facultad de Educación y al Centro de Lecturas, Escrituras y Oralidades (CLEO), de la Universidad de Antioquia, que tornaron posible esta publicación. lectura y escritura; y iv) identificar qué tendencias se abren en torno a la comprensión de prácticas de literacidad.

El deseo que deriva en esta publicación tiene sus raíces en las experiencias de investigación doctoral de las editoras, ${ }^{2}$ así como de la coordinación de la mesa redonda Universidad y literacidad. Experiencias de estudiantes latinoamericanos en torno a prácticas de lectura y escritura académica, en el IV Writing Research Across Borders (WRAB) / Investigación en Escritura a través de las Fronteras, realizado en la Pontificia Universidad Javeriana, sede Bogotá, en febrero de 2017. Durante este evento, investigadores expusieron sus trabajos sobre tendencias y perspectivas de estudio sobre el tema del lenguaje, la formación, la lectura y la escritura académicas. En la línea "Experiencias y representaciones de los estudiantes”, se organizó la mesa redonda en torno a la pregunta: ¿cómo viven los estudiantes la academia a través de sus procesos de lectura y escritura? La riqueza de los aportes puestos en común en la mesa redonda y el panorama de la investigación en Latinoamérica que los mismos presentaron llevaron a las editoras a invitar a la revista Íkala a dedicar este número temático a los estudios de literacidad.

Para comprender el modelo, se presenta a continuación una aproximación general a la concepción del lenguaje en los NLs y se señalan algunas publicaciones que han sido hitos en el contexto latinoamericano. También se plantean algunas líneas de investigación que han sido fructíferas en el estudio de los NLS, de las cuales emergen la mayoría de los artículos que integran este número y que, a su vez, demarcan retos y posibilidades para seguir pensando las prácticas de alfabetización en el campo educativo en la región.

2 Que derivaron en las tesis "Escritas afirmativas: estratégias criativas para subverter a colonialidade em trajetórias de letramento acadêmico" (2016) de Luanda Sito, y "La lectura en la formación universitaria. Análisis de prácticas de enseñanza y aprendizaje de lectores de campos disciplinares específicos" (2018) de Emilce Moreno. 


\section{Marco de comprensión del lenguaje y la literacidad}

En los trabajos que componen este número hay el propósito de discutir el impacto de los NLS (Heath, 1982; Street, 1984, 1993; Barton, 1994; Kleiman, 1995, 2010; Zavala, Niño-Murcia y Ames, 2004), en áreas como la educación, los estudios de lenguaje y las ciencias sociales, entre otros. En este campo "se estudian las prácticas de alfabetización en ámbitos culturales específicos, pasando de lo universal a lo particular con la ayuda de métodos etnográficos" (Zavala et al., 2004, p. 9). Los NLS, como perspectiva epistemológica crítica, se oponen al enfoque psicológico tradicional de la literacidad, es decir, en el que "el procesamiento mental" y "lo cognitivo" juegan un papel primordial para los lectores y escritores, asociado en especial a la recuperación y a la decodificación de la información. Desde este lugar, se argumenta que las prácticas letradas han de ser estudiadas de modo integrado, como lo plantea Gee (2015):

Esa es la razón por la que los NLS tendieron a estudiar no la literacidad en sí misma directamente, sino temas como «sistemas de actividad» (Engeström 1987); «discursos» (Gee 2011 [1990], 2014 [1999]); «discurso en comunidades» (Bizzell 1992); «Culturas» (Street, 1995); «Comunidades de prácticas» (Lave y Wenger 1991; Wenger 1998); «actor-actante y redes» (Latour 2005); «Colectivos» (Latour, 2004); «Grupos de afinidad» o «espacios de afinidad» (Gee 2004): los nombres difieren y hay otros, pero todos son nombres de formas en que las personas se organizan socioculturalmente para participar en actividades (citados por Gee, 2015, p. 36).

Desde esta perspectiva epistemológica, se observan y se analizan prácticas situadas de lectura y escritura articuladas al mundo social (escuela, universidad, trabajo, iglesia, comunidad, etc.), a partir de determinadas formas de actuar, sentir, creer y pensar. Es decir, se estudian las prácticas de literacidad en diferentes contextos culturales en relación a cómo se participa y cómo se usan los textos; se hace una aproximación a las prácticas letradas que involucran la acción, la interacción, los valores y las tecnologías. Igualmente, se preocupa por estudiar aquellas prácticas que son marginalizadas e invisibilizadas, de manera que se identifiquen relaciones de poder, subjetividades y modos de agencia que marcan las formas de uso del lenguaje en diferentes contextos socioculturales. En esa línea de ideas, se estudian las prácticas sociales mediadas por el lenguaje escrito, con la intención de superar la visión del déficit lingüístico y cultural.

Los Nuevos Estudios de Literacidad obedecen a la traducción del término New Literacy Studies, un campo que emergió en el escenario anglosajón como un giro social a los estudios sobre la alfabetización (literacy, en inglés), conforme a la discusión de Gee (2004) y Street (1993). Mientras en inglés el término literacy se refiere tanto a la alfabetización como a la cultura escrita; en portugués se acuñó el término letramento, y en español, los términos literacidad y cultura escrita. No se trata de un simple cambio terminológico, como puede observarse en la siguiente cita:

\footnotetext{
los avances en la teoría y la investigación sobre la lengua escrita que desde los años 80 del siglo xx aportaron conocimiento que movió a los investigadores a sustituir el concepto de alfabetización por el de literacidad (Heath, 1983; Gee, 1991, 1996; Street, 1984; Barton, 1994; Barton, Hamilton e Ivaničc, 2000). La diferencia principal es que alfabetización se entendió históricamente como la acción de enseñar el alfabeto a los adultos analfabetos o a los niños prealfabetizados, mientras que literacidad o cultura escrita se entiende como las prácticas sociales mediadas por el lenguaje escrito (Hernández, 2016, p. 21).
}

Este campo de estudios, que se ha venido consolidando en América Latina desde la década de los 90, indaga por la cultura escrita y aborda la forma como las personas se apropian de la escritura, preguntándose cuáles son los contextos en que el uso de la escritura ocurre, investigando los procesos interaccionales, o eventos letrados, entre los sujetos. En Brasil, el campo de estudos do letramento ha sido muy proficuo en investigaciones desde la educación y la lingüística aplicada. Allí el concepto de letramento fue creado para traducir el concepto de literacy. Entre las obras clásicas del campo está el libro Os significados do letramento de Angela Kleiman (1995), cuyas 
temáticas reflejan la agenda del campo en el país, con temas como formación docente, literacidad escolar, analfabetismo y tensiones entre prácticas letradas vernáculas y dominantes.

En países hispanohablantes, la vertiente sociocultural de los estudios de literacidad empezó a ganar espacio a partir del año 2000. Escritura y sociedad. Nuevas perspectivas teóricas y etnográficas, coordinada por Virginia Zavala, Mercedes Niño-Murcia y Patricia Ames (2004), logró compilar y traducir estudios seminales del campo — con autores como Brian Street, Shirley Heath y David Barton-; así mismo, estudios etnográficos desarrollados en Perú permitieron conocer un panorama de investigaciones y sus principales referentes conceptuales y metodológicos. Otra obra que presenta un mapeo de investigaciones sobre la cultura escrita en nuestro continente es el libro Lectura, escritura y matemáticas como prácticas sociales, coordinado por Judith Kalman y Brian Street (2009), el cual presenta un buen mapeo de las investigaciones latinoamericanas desde los NLS. Esta obra muestra diferentes estudios en torno al campo de culturas escritas y el sello teórico y metodológico impreso. Desde esta perspectiva sociocultural, también se encuentra el libro Para ser letrados. Voces y miradas sobre la lectura, compilado por Daniel Cassany (2009) en el que se plantean diferentes propuestas sobre la enseñanza de la lectura en el aula, lo cual implica entender que leer es una actividad vinculada a instituciones e ideologías.

Desde este lugar epistemológico en la comprensión del lenguaje, el punto de partida ya no es el texto en sí, sino la gran variedad cultural de las prácticas sociales que lo rodean y lo constituyen, y, en esa medida, no es posible hablar de una sola literacidad (Street 1993, p. 81). Al reconocer la diversidad de estas prácticas letradas no se descuidan las relaciones de poder que atraviesan el uso del lenguaje, marcando las prácticas letradas por divisiones sociales (como género, sexualidad, etnicidad y raza, identidad, entre otras) que resultan en desigualdad. Además, las nociones de literacidades dominantes y vernáculas ayudan en esa descripción, al observar cómo "las primeras son apoyadas por organizaciones poderosas, como la educación, mientras que las otras son menos valoradas y no reciben apoyo de dichas instituciones" (Barton y Hamilton, 1998, p. 118). De ahí que sea pertinente el estudio etnográfico de literacidades locales, desde perspectivas críticas, la gran apuesta de los NLS. En su gran mayoría, son estudios que están vinculados al paradigma cualitativo-interpretativista, y se orientan para un enfoque interdisciplinario, especialmente por medio de abordajes etnográficos y discursivos, con concepciones sociales de lenguaje, como el dialogismo bajtiniano o la sociolingüística.

En definitiva, el modelo de las literacidades plantea una posición crítica sobre las prácticas letradas en contextos situados, que no pueden disociarse de las relaciones de poder, la construcción de identidad, las culturas escritas, incluidas las mediadas por tecnologías digitales, y que están determinadas por la disciplina, la historia y la ideología.

\section{Retos y oportunidades de los NLS}

La investigación de las prácticas de lenguaje (orales, escritas, multimodales) desde los NLs requiere que su estudio se realice en la vida social, atendiendo al contexto cultural mismo. De ahí que resulte importante en el marco educativo la variación y los usos lingüísticos de los estudiantes, así como los contextos de aprendizaje como escenarios fundamentales de estudio. Sin embargo, la investigación y la reflexión sobre el lenguaje y las prácticas de literacidad desde esta perspectiva suponen desafíos, dado que los profesores, directivos docentes y aquellos que diseñan los currículos se ven retados a asumir una postura social y crítica de las prácticas de enseñanza y aprendizaje, las prácticas de lenguaje (oral, escrito, multimodal), los discursos imperantes, los roles de los actores educativos, y las políticas. Sobre este tema, Street (1997) plantea algunos principios en los cuales basa la aplicación de los NLS a la educación en general y a las prácticas de literacidad en particular:

a. La literacidad es más compleja que lo que el plan de estudio y la evaluación actuales permiten. 
b. Los planes de estudio y la evaluación que reducen la literacidad a habilidades simples y mecanicistas no hacen justicia a la riqueza y complejidad de las prácticas reales de alfabetización en la vida de las personas.

c. Si queremos que los estudiantes desarrollen y mejoren la riqueza y la complejidad de las prácticas de literacidad evidentes en la sociedad en general, entonces, necesitamos planes de estudio y evaluaciones que sean en sí mismos ricos y complejos y se basen en la investigación de prácticas reales.

d. Para desarrollar planes de estudio y evaluaciones ricos y complejos en torno a prácticas de literacidad, necesitamos modelos de alfabetización y de pedagogía que capturen la riqueza y la complejidad de las prácticas reales (p. 53).

De acuerdo a lo anterior, la riqueza y las posibilidades de las prácticas de literacidad y los usos del lenguaje reales en diferentes contextos plantean desafíos y oportunidades para los investigadores y los actores educativos, dado que las prácticas extraescolares también dialogan con los procesos formativos, y finalmente las formas de acceso al conocimiento, los modos de leer y escribir se arraigan a concepciones, valores, ideologías, identidades, relaciones de poder que no se pueden desconocer.

Dado lo anterior, en el contexto geopolítico e histórico latinoamericano, los estudios de literacidades plantean una epistemología para pensar las prácticas de enseñanza del lenguaje en un sentido amplio, poniendo el acento en el carácter heterogéneo de los usos orales, escritos y multimodales a través de su conceptualización como prácticas sociales y dialógicas. De acuerdo a los estudios que se incluyen en este número, se señalan a continuación áreas que han sido tradicionalmente abordadas por los estudios de literacidades y otras que requieren mayor consideración teórica e investigación empírica.

\section{La comprensión de las prácticas de lectura y escritura en la educación de jóvenes y adultos}

En el abordaje de este tipo de estudios se requiere "encontrar el eslabón perdido del cambio cognitivo adulto" (Scribner, 1997, p. 206). En esa medida, se abre una gama de posibilidades de estudio de usos de la literacidad en diferentes contextos (religioso, laboral, doméstico, comunitario, escolar, etc.), así como los modos de ponerlos en diálogo desde el currículo escolar. En esta temática se incluye el artículo "Aportes de los Nuevos Estudios de Literacidad al campo de la educación de jóvenes y adultos", de María del Carmen Lorenzatti, Gladys Blazich y Rocío Arrieta.

\section{Prácticas de lectura y escritura en contextos rurales}

Aún resulta pertinente la pregunta ¿qué dificultades enfrenta el aprendizaje de la lectura y la escritura en el campo? Una explicación inicial se relaciona con la pobre infraestructura y los escasos materiales educativos de estas escuelas, así como la débil articulación de la familia y la escuela y la diversidad lingüística y sociocultural de la población. En estos estudios, resulta importante el análisis de la dicotomía alfabetismo/analfabetismo en marcos históricos y económicos rurales específicos, que evidencien aquello que puede dificultar o potenciar la construcción de conocimiento y la participación social a través de las prácticas letradas. Para ilustrar este tema se encuentra el artículo "Estrategias migratorias, reconfiguraciones de identidades campesinas y participación en la cultura escrita”, de Elisa Cragnolino.

\section{Aporte de la perspectiva etnográfica a la comprensión de las prácticas letradas}

Desde los NLS, adoptar una postura etnográfica permite comprender las prácticas de literacidad en el marco social e institucional en el cual las personas usan los recursos semióticos y textuales para desarrollar funciones concretas y para entender cómo se comunican en contextos escolares y académicos. Al contrario de enfoques cognitivos, la etnografía como ideología y teoría permite abordar las desigualdades (Blommaert, 2005). Al respecto, Street (2009) plantea que una perspectiva etnográfica sobre las prácticas letradas y la comprensión de las desigualdades "puede sensibilizarnos en relación 
con las formas en las que el poder de nombrar y definir constituye un componente crucial de la desigualdad" (p. 84). Por ejemplo, entender las culturas escritas, atendiendo a sus significados locales y superando la visión deficitaria de la escritura tan instalada en las prácticas de enseñanza; $\mathrm{o}$, a través de la etnografía, describir y analizar los usos de los textos escritos en contextos situados. En la compilación "Aprendizaje, cultura y desarrollo. Una aproximación interdisciplinaria”, de Frisancho, Moreno, Ruiz y Zavala (2011), reseñado en este número, se incluyen trabajos de esta naturaleza. También ilustra este tema el artículo "Desafios éticos e metodológicos em pesquisas sobre letramento do professor: provocações em busca de novos caminhos", de Paula Baracat De Grande, Marília Curado Valsechi y Carolina Assis Dias Vianna.

\section{Construcción identitaria en el marco educativo}

Las construcciones sociales de raza y racismo tienen efectos reales en las prácticas sociales y en las de literacidad, especialmente por el poder opresivo que ejerce el discurso racial (Bailey, 2000). Las prácticas letradas en el ámbito escolar tienen la posibilidad de reafirmar o no, reconocer o no los conflictos de poder que atraviesan estas prácticas, sea en relación con la identidad racial u otras, como identidades sexuales, de género, de edad, región, entre otras. Específicamente, en este tema, se encuentra el artículo "A construção de identidades étnico-racial em eventos de letramento numa escola pública municipal de São Paulo", de Claudia Lemos Vóvio, Estevão Armada Firmino.

\section{Reconocimiento de las perspectivas decoloniales y crítica del lenguaje}

En el marco dela teoría crítica, la visión tradicionalde cultura da paso a una visión que enfatiza "el carácter político, social y conflictivo de la misma y la visualiza como el campo de lucha por el acceso a la hegemonía (Castro-Gómez, citado por Frisancho et al., 2011, p. 13). Se trata de entender las prácticas letradas en ámbitos de tensión, de desigualdad, de conflicto o en contextos minoritarios (p. e. estudiantes indígenas o afrodescendientes que ingresan a la universidad, estudiantes de básica y secundaria en contextos vulnerables por situación de desplazamiento o emigración). Para ilustrar la necesidad de reconocer la perspectiva crítica del lenguaje - clave para entender las prácticas de literacidad - se encuentra el artículo de Virginia Zavala, titulado: "Justicia sociolingüística para los tiempos de hoy". Desde el giro decolonial, se pueden establecer diálogos con los estudios de literacidad, que permitan entender las prácticas y los discursos en torno a la forma en que la cultura escrita se establece en la sociedad. Se trata de plantear opciones creativas y emancipadoras de acompañar las prácticas letradas. En esta línea de pensamiento está el artículo "De los Nuevos Estudios de Literacidad a las perspectivas decoloniales en la investigación sobre literacidad" de Gregorio Hernández-Zamora.

\section{Formación de maestros, programas de escritura y literacidad}

Aquí se inscriben aquellas iniciativas que contribuyen a la formación, empoderamiento y reflexión de los profesores en torno a sus prácticas de enseñanza y permiten ver cómo estas se ligan a usos del lenguaje reales instalados en la vida de las personas. En este campo resulta importante la reflexión sobre cómo imperan prácticas de enseñanza desde el "modelo autónomo" (Street, 1993, 1995, 2005), es decir, basado en el supuesto de que las prácticas letradas en sí mismas tienen efectos en otras prácticas sociales y cognitivas. Sin embargo, las formas como los profesores y sus estudiantes interactúan ya es una práctica social que afecta los procesos de acceso a la cultura escrita, de ahí que no sea válido sugerir que estos procesos se dan de modo neutral y universal. Por el contrario, el "modelo ideológico" enfatiza "no sólo en los significados culturales, sino también la dimensión de poder de los procesos de lectura y escritura" (Street, 2005, p. 418). En esa medida, estos procesos no solo varían de acuerdo al contexto social, a las normas y los discursos en relación con la identidad, el género y las creencias, sino en cuanto a los usos y significados que se insertan en relaciones de poder. En la parte formativa, también se destaca el aporte 
que se puede hacer desde teorías que dialogan con los NLS: análisis crítico del discurso, la sociolingüística, la antropología cultural, los estudios culturales, las pedagogías críticas, las teorías decoloniales, las teorías críticas, etc. Los artículos que ilustran este tema son: "Literacidad e identidades en las investigaciones sobre formación docente en Brasil", de Angela B. Kleiman, y "Desafios éticos e metodológicos em pesquisas sobre letramento do professor: provocações em busca de novos caminhos", de Paula Baracat De Grande, Marília Curado Valsechi y Carolina Assis Dias Vianna.

\section{Literacidades académicas}

La noción de literacidad académica se asocia a la participación de los sujetos en sus culturas escritas, en prácticas discursivas académicas articuladas a los procesos de enseñanza, aprendizaje e investigación, las cuales involucran el acceso, la construcción, la producción, la divulgación y la apropiación de conocimiento a partir de prácticas comunicativas orales, escritas y multimediadas. $\mathrm{Al}$ respecto, Hernández explica:

La literacidad académica es una práctica situada en contextos sociales e institucionales específicos (la educación superior y la investigación científica, sus comunidades y prácticas), por lo que no es algo que los alumnos deban poseer al ingresar a la universidad, sino que es tarea de la universidad socializarlos en dichas prácticas (Carlino, 2005, citado por Hernández, 2016, p. 19).

Subtemas asociados a esta categoría se relacionan con modos de aprendizaje de los géneros académicos, convenciones retóricas dominantes, identidad y manejo de la voz del autor, etc. En este caso, se presentan artículos que reconocen los géneros discursivos contextualizados como formas de acción social; estos son: "Lo que escriben los científicos locales. El texto como acción social”, de Roberto Méndez-Arreola y Judith Kalman, y también "La escritura de géneros especializados y su relación con la identidad disciplinar. Estudio de caso en Ingeniería Industrial”, de Emilce Moreno.
En relación con las dos últimas áreas, están los programas de escritura pues, como lo plantea Denny (2010), los centros o programas de escritura pueden constituirse como "sitios para el activismo y el cambio social” (p. 26), y, contribuir así a la desnaturalización de prácticas racistas, transformando la práctica escritural en escenarios más inclusivos. Este tipo de iniciativas institucionales en el ámbito universitario se implementan con el fin de mejorar las competencias de escritura de los estudiantes y pueden, a su vez, configurar escenarios que potencien la reflexión sobre el papel del lenguaje en la vida académica, y contribuyan a la desnaturalización de prácticas prescriptivas de enseñanza de la escritura, al afianzamiento de una política de alfabetización inclusiva y al reconocimiento de diferentes estilos y formas de escritura, más allá de la académica o de concepciones normativas y de "uso correcto" de la escritura. Es ejemplo de ello el artículo "LEO en la práctica: la experiencia formativa en un Centro de Lecturas, Escrituras y Oralidades”, de Luanda Rejane Soares Sito, Leidy Yaneth Vásquez Ramírez y Juan Camilo Méndez Rendón.

\section{Tecnologías digitales y literacidad}

Si bien los estados de Latinoamérica han invertido en las últimas dos décadas en equipamiento y formación docente en tecnologías digitales, aún estas tecnologías se encuentran subutilizadas especialmente en las aulas (Lugo, López y Toranzos, 2014). De acuerdo con ello, todavía está pendiente en la agenda lo relacionado con el acceso a una educación de calidad de mayor alcance, lo cual se debe a déficits en infraestructura o conectividad, a la tensión entre cultura digital, conocimiento y educación y a la necesidad de formación y desarrollo profesional en el manejo de Tecnologías de la Información y la Comunicación (TIC) de los docentes y directivos. En esa línea, se plantea el "giro digital" en el cual:

No se trata solo de reconocer e investigar la proliferación de los dispositivos, los servicios y las redes en la vida cotidiana de muchas personas en todo el mundo, sino también de incluir un interés académico sostenido para desarrollar formas de entender los cambios que la gente hace y los nuevos usos relacionados con la 
manera en la que comparte y retoma los significados y los recursos mediados o producidos de manera digital (Runnel et al.; Mills, citados por Knobel y Kalman, 2017, pp. 21-22).

Este giro ligado a los estudios de literacidades permite enriquecer la discusión sobre los usos de las tecnologías digitales en las prácticas de enseñanza y aprendizaje. Además, abre preguntas sobre cómo los lenguajes multimodales, los dispositivos tecnológicos y las redes digitales generan posibilidades de trabajo y de formación docente que pueden tener un sentido pedagógico y no instrumental. En este tema no se recibieron artículos, pero se incluyó la reseña del libro Aprendizaje docente y nuevas prácticas de lenguaje. Posibilidades de formación en el giro digital, editado por Knobel y Kalman (2017).

\section{Lenguas indígenas, políticas de bilingüismo y multilingüismo}

Si bien en este número no se recibieron artículos de estos temas en la perspectiva de los NLS, hay una preocupación por entender qué enfoques educativos y lingüísticos favorecen la enseñanza de poblaciones indígenas, así como el fortalecimiento de las lenguas maternas, segundas lenguas o lenguas adicionales. De otro lado, ha sido focalizado también el estudio de comunidades bilingües y multilingües y sus programas de alfabetización en diferentes lugares del mundo, incluida América Latina. También existe el interés por analizar prácticas de lectura y escritura en contextos de escritura escolar y profesional en lengua extranjera (Lillis y Curry, 2006; Canagarajah, 2002a, 2002b). Como ejemplos, adicionales podemos citar los trabajos de Maher (1998, 2007), Hornberger (2001), Zavala et al. (2004), Zavala (2010), Zavala y Córdoba (2010) y Soler (2013).

Sin ánimo de reducir las líneas de acción de los estudios de literacidades, se han planteado algunos posibles focos de trabajo para continuar pensando la investigación y las prácticas de enseñanza, o seguir revisando los discursos positivistas y prescriptivos en torno al lenguaje, así como, las posibilidades de influir en el diseño curricular de áreas como lenguaje desde una perspectiva más interdisciplinar y crítica.

\section{Conclusiones}

Con esta esta publicación, se mostraron algunos ejemplos de lo que se viene desarrollando en investigación sobre literacidades, en América Latina. En este sentido, los artículos permiten avizorar temas, preguntas y conceptos, escenarios, retos que son relevantes en el campo. Cuestiones como la educación de jóvenes y adultos, la formación docente, la migración, las identidades, el género discursivo, las escrituras especializadas, las comunidades (campesinas, palenqueras, quilombolas, etc.) y las literacidades académicas hacen parte de la agenda de investigación desde este marco, mientras nociones como justicia sociolingüística y teoría decolonial vienen ganando espacio entre los investigadores.

También es importante destacar que hay temas que no están representados, como los trabajos sobre culturas escritas mediadas por tecnologías digitales y multimodalidad, los cuales tienen mucha fuerza y se reconocen dentro de la categoría de "literacidades digitales". Efectivamente, la tecnología se ha constituido en un factor estructurante de las prácticas letradas. De otro lado, existen campos en los cuales se investiga en la actualidad desde los estudios de literacidades que, aunque no fueron documentados, muestran la amplia gama de posibilidades de trabajo en cuanto a la diversidad de culturas escritas en América Latina, tales como: la biliteracidad (leer y escribir en dos lenguas), influencias ecológicas (familia, amigos, etc.) en el estudio de las prácticas de literacidad o biliteracidad, comunidades de práctica, los videojuegos, espacios virtuales y el aprendizaje en el siglo XXI, estudios etnográficos de escritura en los distintos niveles escolares y en escenarios no escolares, entre otros.

A través de la lectura de este número se pretenden identificar, en el contexto latinoamericano, las tendencias que se tejen actualmente en torno a la comprensión de las prácticas de literacidad, así como conocer y revisar los aportes de la perspectiva para la investigación sobre prácticas de lectura 
y escritura en los diferentes niveles educativos. Los estudios que se incluyeron evidencian marcos teóricos, hallazgos de investigaciones, análisis, reflexiones sobre culturas escritas de diferentes lugares de la región, los cuales entran en diálogo con debates y discusiones teóricas y metodológicas a nivel global. Efectivamente, los Nuevos Estudios de Literacidad o Nuevos Estudios de Cultura Escrita "se constituyen en una epistemología crítica sobre las prácticas letradas contemporáneas, la cual reconoce además que la literacidad crítica sirve para analizar, criticar, rediseñar y transformar las estructuras sociales que influyen en la vida de las personas" (Rogers y O’Daniels, citado por Vargas-Franco, 2018, p. 157).

Adicionalmente, en nuestro contexto, como lo plantea Jouve-Martín, podríamos hablar de un Latin American Literacy Studies, pues para el autor los estudios sobre literacidad en América Latina no son apenas una forma de "catalogar eventos letrados concretos dentro de prácticas de escritura específicas, sino que forman parte de un movimiento intelectual más amplio, que critica los modelos que han guiado el desarrollo intelectual, económico y político del continente" (Jouve-Martín, 2009, p. 395), enfocando temas como escritura, hegemonía, hibridación y subalternidad. De ahí la importancia de que, desde las prácticas sociales mediadas por el lenguaje escrito y la enseñanza de la lectura, la escritura y la oralidad, se busque superar la visión del déficit lingüístico y cultural y crear escenarios de empoderamiento y justicia social en el marco de lo educativo.

El contexto latinoamericano plantea excelentes escenarios y posibilidades para explorar las líneas de trabajo enunciadas anteriormente, pues el marco epistemológico de los estudios de literacidades permiten diseñar programas, plantear pedagogías y políticas lingüísticas y educativas más acordes con las culturas escritas y a los diferentes problemas sociales y políticos que inciden en el acceso, la adquisición y la distribución desigual de las prácticas de lenguaje (oral, escrito y multimodal) en la región.

\section{Referencias}

Barton, D. (1994). The social impact of literacy. En L. Verhoeven (Ed.), Functional literacy. Theoretical issues and educational implications (pp. 185-197). Amsterdam: John Benjamins.

Barton, D. y Hamilton, M. (1998). Local literacies. Reading and writing in one community. Londres: Routledge.

Bailey, B. (2000). Language and negotiation of racial/ethnic identity among Dominican Americans. Language in Society, 29, 555-582.

Blommaert, J. (2005). Discourse. A critical introduction. Cambridge: Cambridge University Press.

Canagarajah, A. S. (2002a). Critical academic writing and multilingual students. Ann Arbor: University of Michigan Press.

Canagarajah, A. S. (2002b). A geopolitics of academic writing. Pittsburgh: University of Pittsburgh Press.

Cassany, D. (Comp.) (2009). Para ser letrados. Voces y miradas sobre la lectura. Barcelona: Paidós Educador.

Denny, H. (2010). Facing the center: Toward an identity politics of one-to-one mentoring. Logan, UT: Utah State UP.

Frisancho, S., Moreno, M. T., Ruíz, P. y Zavala, V. (Eds.). Aprendizaje, cultura y desarrollo. Una aproximación interdisciplinaria. Lima: Fondo Editorial Pontificia Universidad Católica del Perú.

Gee, J. P. (2015). The New Literacy Studies. En J. Rowsell y K. Pahl (Eds.), The Routledge handbook ofliteracy studies (pp. 35-48). Londres-Nueva York: Routledge.

Gee, J. P. (2004). Oralidad y literacidad: de Elpensamiento salvaje a Ways with words. En V.Zavala, M. Niño-Murcia, y P. Ames (Eds.), Escritura y sociedad. Nuevas perspectivas teóricas y etnográficas (pp. 23-55). Lima: Red para el Desarrollo de las Ciencias Sociales en el Perú.

Heath, S. B. (1982). What no bedtime story means: narrative skills at home and school. Language and Society, 11(1), 49-76.

Hernández, G. (2016). Literacidad académica. Ciudad de México: Universidad Autónoma Metropolitana.

Hornberger, N. (2001). Criando contextos eficazes de aprendizagem para o letramento bilíngüe. En M. I. Pagliarini y A. A. Assis-Peterson (Orgs.), Cenas de sala de aula (pp. 23-50). Campinas, sp: Mercado de Letras.

Jouve-Martín, J. R. (2009). Escritura, hegemonia y subalternidad: de los New Literacy Studies (NLS) a los Latin American Literacy Studies (LALS), and back. En J. Kalman y B. V. Street, (Coords.), Lectura, escritura y 
matemáticas como prácticas sociales: diálogos con América Latina (pp. 387-398). México: Siglo XXI.

Kalman, J. y Street, B. (Coord.). (2009). Lectura, escritura y matemáticas como prácticas sociales. Diálogos con América Latina. Ciudad de México: Siglo XXI.

Knobel, M. y Kalman, J. (coord.) (2017). Aprendizaje docente y nuevas prácticas de lenguaje. Posibilidades de formación en el giro digital. Ciudad de México: SM.

Kleiman, A. B. (org.) (1995). Os significados do letramento: uma nova perspectiva sobre a prática social da escrita. Campinas, SP: Mercado Aberto.

Kleiman, A. B. (2010). Trajetórias de acesso ao mundo da escrita: relevância das práticas não escolares de letramento para o letramento escolar. Perspectiva, 28(2), 375-400, Florianópolis.

Lillis, T. y Curry, M. J. (2006). Professional academic writing by multilingual scholars: interactions with literacy brokers in the production of English medium texts. Written Communication, 23(1), 3-35.

Lugo, M. T., López, N. y Toranzos, L. (2014). Informe sobre tendencias sociales y educativas en América Latina. Politicas TIC en los sistemas educativos de América Latina. Buenos Aires: UNEsCo.

Maher, T. M. (2007). A educação do entorno para a interculturalidade e o plurilinguismo. En A. B. Kleiman y M. C. Cavalcanti (Orgs.), Linguística aplicada: suas faces e interfaces (pp. 255-270). Campinas, SP: Mercado de Letras.

Maher, T. M. (1998). Sendo índio em português. En I. Signorigni (Org.), Lingua(gem) e identidade: elementos para uma discussão no campo aplicado (pp. 115-138). Campinas, SP: Mercado de Letras; FAPESP; FAEP/UNICAMP.

Moreno, E. (2018). La lectura en la formación universitaria. Análisis de prácticas de enseñanza y aprendizaje de lectores de campos disciplinares especificos. Tesis doctoral inédita, Universidad Pedagógica Nacional, Bogotá.

Scribner, S. (1997 [1985]). Vygotsky's uses of history. En T. Ethel et al. (Eds.), Mind and social practice: Selected writings of Sylvia Scribner (pp. 241-265). Cambridge: Cambridge UP.

Sito, L. (2016). Escritas afirmativas: estratégias criativas para subverter a colonialidade em trajetórias de letramento acadêmico. Tesis doctoral inédita, Universidad Estatal de Campinas, Campinas, sp, Brasil.
Soler, S. (2013). Usted ya en la universidad y no saber escribir. Escritura y poder en la universidad. Bogotá: Universidad Distrital Francisco José de Caldas.

Street, B. (1984). Literacy in theory and practice. Cambridge: Cambridge University Press.

Street, B. (1993). Introduction: The New Literacy Studies. En B. V. Street (Ed.), Crosscultural approaches to litera$c y$ (pp. 1-21). New York: Cambridge University Press.

Street, B. (1995). Social literacies: critical approaches to literacy in development, ethnography and education. Nueva York: Longman Publishing.

Street, B. (1997). The implications of the 'New Literacy Studies' for Literacy Education. English in Education, 31(3), p. 45-59.

Street, B. (2005). Recent applications of New Literacy Studies in educational contexts. Research in the Teaching of English, 39(4), 417-423.

Street, B. (2009). "Perspectivas etnográficas y políticas sobre cultura escrita: el poder de nombrar y definir". En J. Kalman, y B. Street, Lectura, escritura y matemáticas como prácticas sociales: diálogos con América latina (pp. 84-98). México: Siglo XXI-CrEFAL.

Vargas-Franco, A. (2018). Nuevos estudios de cultura escrita y educación: implicaciones para la enseñanza del lenguaje en Colombia. Perspectiva Educacional, 57(3), 153-174. https://dx.doi. org/10.4151/07189729-vol.57-iss.3-art.757

Zavala, V. (2011). La escritura académica y la agencia de los sujetos. Cuaderno Comillas, 1, 52-66.

Zavala, V. (2010). Quem está dizendo isso?: letramento acadêmico, identidade e poder no ensino superior. En C. L. Vóvio, L. S. Sito y P. B. de Grande, Letramentos: rupturas, deslocamentos e repercussóes de pesquisas em Linguistica Aplicada (pp. 71-95). Campinas, SP: Mercado de Letras.

Zavala, V.; y Córdova, G. (Org.). (2010). Decir y callar. Lenguaje, equidad y poder en la universidad peruana. Lima: Editorial de la Pontificia Universidad Católica del Perú.

Zavala, V.; Niño-Murcia, M. y Ames, P. (Eds.). (2004). Escritura y sociedad. Nuevas perspectivas teóricas y etnográficas. Lima: Red para el Desarrollo de las Ciencias Sociales en el Perú.

How to reference this article: Moreno, E. y Sito, L. (2019). Discusiones actuales, oportunidades y horizontes en los estudios sobre literacidades en América Latina. Íkala, Revista de Lenguaje y Cultura, 24(2), 219-229. Dor: 10.17533/udea.ikala.v24n02a02 